\title{
Itinéraires
}

Itinéraires Littérature, textes, cultures

2012-1 | 2012

Genres et avant-gardes

\section{Biographie et psychanalyse des femmes surréalistes}

\section{Georgiana Colvile}

\section{Q OpenEdition}

1 Journals

\section{Édition électronique}

URL : http://journals.openedition.org/itineraires/1290

DOI : $10.4000 /$ itineraires. 1290

ISSN : 2427-920X

Éditeur

Pléiade

\section{Édition imprimée}

Date de publication : 1 septembre 2012

Pagination : 123-135

ISBN : 978-2-296-55776-5

ISSN : 2100-1340

Référence électronique

Georgiana Colvile, "Biographie et psychanalyse des femmes surréalistes », Itinéraires [En ligne], 2012-1 | 2012, mis en ligne le 01 septembre 2012, consulté le 30 avril 2019. URL : http://

journals.openedition.org/itineraires/1290; DOI : 10.4000/itineraires.1290

\section{(a) $\oplus \Theta \Theta$}

Itinéraires est mis à disposition selon les termes de la licence Creative Commons Attribution - Pas d'Utilisation Commerciale - Pas de Modification 4.0 International. 


\title{
Biographie et psychanalyse des femmes surréalistes
}

\begin{abstract}
This essay explores the relevance of biography and psychoanalysis as hermeneutic approaches to the literary and plastic production of women surrealists. Women were usually marginalized within the movement(s), and used their creativity to achieve an autonomous identity. The result was a proliferation of self expression, mainly autobiographies and self-portraits. Their lives, desires and traumas mirror their art, so biography and psychoanalysis provide a necessary point of departure for other theoretical investigations. Colvile concentrates on texts by Claude Cahun and Leonora Carrington.
\end{abstract}

Keywords : biography, psychoanalysis, women surrealists, self-expression, mirror Mots clés : biographie, psychanalyse, femmes surréalistes, auto-expression, miroir

« [C]haque mot écrit par Unica [Zürn] est autobiographique ${ }^{1}$. »

« Tout récit ne ramène-t-il pas à l'CEdipe? Raconter, n'est-ce pas toujours chercher son origine, dire ses démêlés avec la Loi, entrer dans la dialectique de l'attendrissement et de la haine ${ }^{2}$ ? »

Lorsque Montaigne écrivait de sa tour d'ivoire : «je suis moy-mesmes la matiere de mon $\operatorname{livre}^{3} »$, s'engageant à dire la vérité, il relevait en prolepse le défi du «pacte autobiographique» de Philippe Lejeune ${ }^{4}$. Le fait de décrire le microcosme familier de son corps et de sa psyché, avant

1. Ruth Henry, « Postface », dans Unica Zürn, Sombre Printemps, trad. française par Ruth Henry et Robert Valençay [1971], Paris et Montréal, Écriture, 1997, p. 107.

2. Roland Barthes, Le Plaisir du texte [1973], repris dans Euvres complètes, t. IV, 1972-1976, Paris, Seuil, 2002, p. 248.

3. Montaigne, " $\mathrm{Au}$ lecteur", Les Essais $[1580 ; 1588 ; 1595]$, repris dans Euvres complètes, Paris, Gallimard, coll. « Bibliothèque de la Pléiade », 1962, p. 9.

4. Voir Philippe Lejeune, Le Pacte autobiographique, Paris, Seuil, coll. «Poétique », 1975. 
d'aborder le macrocosme de l'univers, indique à la fois une certaine modestie et une grande confiance en soi. Plus tard, Freud, au seuil de l'invention de la psychanalyse, entreprit une autoanalyse, utilisant son propre cas comme cobaye, afin de mieux comprendre ses patients. Il pratiqua toute sa vie le rituel autoréférentiel de se raconter sa propre histoire, y puisant toujours de nouveaux schémas psychanalytiques, bien que pas toujours applicables aux femmes. Pourtant, comme le suggère Jacqueline Cosnier :

Si la féminité est restée pour Freud l'énigme, le continent noir, elle n'a cessé de rester une source de sa créativité, justement parce que les représentations auxquelles elle a pu être associée ont partie liée avec les impressions les plus précoces, potentiellement les plus traumatiques, génératrices de scénarios fantasmatiques d'autant plus inquiétants qu'ils prennent pour objet le corps maternel, et sont alors contre-investis ${ }^{5}$.

Un grand nombre de femmes ont adhéré au surréalisme, même si elles restaient souvent en marge et furent longtemps ignorées des critiques. Contrairement à Montaigne, ces femmes écrivains et/ou plasticiennes tissaient leur matière, leur moi ou leur identité à partir de leur propre littérature, peinture, photographie, sculpture, collages, objets, etc. Elles faisaient rarement école entre elles, mais une forte pulsion d'auto-expression s'avère commune à la plupart d'entre elles. Aussi ont-elles produit une quantité phénoménale d'autobiographies, de lettres, fictions, poèmes et pièces de théâtre plus ou moins autobiographiques, ainsi que d'autoportraits peints ou photographiés. Cette production a motivé divers critiques, généralement féminins, à partir de $1977^{6}$, de plus en plus nombreux par la suite, à les révéler au public par des anthologies, biographies ou monographies, dont les études récentes sur Dora Maar, Leonora Carrington, Lee Miller, Nadja, Nusch Éluard et Jacqueline Lamba ${ }^{7}$. Par ailleurs, la plupart de ces femmes ont voulu se recréer une identité propre en racontant leur histoire à la suite d'un trauma (Claude Cahun, Leonora Carrington, Kay Sage, Gisèle Prassinos, Bona, Unica Zürn, Dorothea Tanning, entre autres) et c'est surtout là qu'intervient la psychanalyse.

Ni l'approche biographique, ni la psychanalytique ne sont très prisées dans le domaine de la recherche universitaire actuelle qui, à une époque post-féministe, n'accepte guère non plus l'idée de la marque différenciée

5. Jacqueline Cosnier, Destinées de la féminité, Paris, PUF, coll. « Le fait psychanalytique », 1987, p. 10.

6. Avec le no 14-15 de la revue Obliques sur « La Femme surréaliste ».

7. Mary Ann Caws, Dora Maar with \& without Picasso, Londres, Thames \& Hudson, 2000 ; Susan Aberth, Leonora Carrington, New York, Lund Humphries, 2004; Carolyn Burke, Lee Miller, Londres, Bloomsbury Publishing, 2005; Hester Albach, Leona [Nadja] Héroïne du surréalisme, Arles, Actes Sud, 2009; Chantal Vieuille, Nusch : portrait d'une muse du surréalisme, Paris, Artelittera, 2010; Alba Romano-Pace, Jacqueline Lamba, Paris, Gallimard, coll. « Témoins de l'Art », 2010. 
du genre masculin ou féminin dans l'écriture ou les arts plastiques. Or, c'est de la vie des femmes surréalistes, de leur marginalité par rapport au(x) mouvement(s), ainsi que des rôles de muses, de femmes enfants, de folles, de sorcières, etc., qu'on leur imposait, que provenaient l'altérité et l'aspect spéculaire de leur créativité. Il y a une vingtaine d'années, une critique américaine, Cheryl Walker, a écrit à propos des poètes féminins en général : « Il nous faut tenir compte de la vie d'une poétesse, des livres qu'elle lisait, de la race et de la classe auxquelles elle appartenait, de sa réaction à la ville [...], de son expérience d'un monde déchiré par la guerre, de ses amours ${ }^{8}$. » Whitney Chadwick décrit ces femmes comme " jeunes, belles et rebelles ${ }^{9}$ ", et leurs confrères et/ou conjoints avaient tendance à projeter leurs propres fantasmes poétiques, érotiques, même parfois politiques sur elles, sans attribuer beaucoup d'importance à leur production littéraire et/ou artistique. Leur rébellion ne se dirigeait pas nécessairement contre le surréalisme, mais plutôt contre la société ou leur famille, qui s'érigeaient en obstacles entre elles et la pratique de leur art.

Il me semble qu'en ce qui concerne ces créatrices, le « pacte » devrait être biographique plutôt qu'autobiographique, en d'autres termes il devrait être l'affaire du critique/chercheur et non pas de l'écrivaine/artiste. Il arrive que les deux soient réunis en une seule et même personne, telle la cinéaste/ théoricienne Maya Deren (1917-1961), pour qui, comme le signale Maureen Turim, la forme même d'une œuvre cinématographique relève de la moralité ou de l'idéologie ${ }^{10}$, ou Leonora Carrington, dans un texte unique, En-Bas (1941-1945), auquel je reviendrai. Par contre, chez beaucoup de femmes surréalistes, la forme éclatée de leurs écrits autobiographiques en constitue l'aspect automatique, voire inconscient, proche du stream of consciousness d'un James Joyce ou d'une Virginia Woolf.

Examinons quelques exemples. D'abord Claude Cahun (1894-1954), écrivain et photographe, née Lucy Schwob à Nantes, de père juif libéral, Maurice Schwob, directeur du journal Le Phare de la Loire, frère de l'écrivain symboliste Marcel Schwob, et de mère de famille catholique, conservatrice et antisémite, Mary-Antoinette Courbebaisse. L'étrange récit autobiographique de jeunesse de Cahun, Aveux non avenus, écrit entre 1919 et 1928, illustré de photomontages réalisés en 1928 avec sa compagne Suzanne Malherbe, alias Marcel Moore, publié en 1930 aux Éditions du Carrefour, constitue un véritable collage littéraire, tant la forme en est fragmentaire et diversifiée. Les mots d'ouverture, «L'aventure invisible »,

8. Cheryl Walker, Masks Outrageous and Austere: Psyche and Persona in Modern Women Poets, Bloomington, Indiana University Press, 1991. Ma traduction.

9. Whitney Chadwick, Les Femmes dans le mouvement surréaliste [1985], Paris, Thames \& Hudson, 2002, p. 9.

10. Voir Maureen Turim, "The Ethics of Form », dans Bill Nichols (dir.), Maya Deren \& the American Avant-Garde, Berkeley, Los Angeles, Londres, University of California Press, 2001, p. 78. 
et la revendication initiale du narrateur androgyne de « [n]e voyager qu'à la proue de moi-même », annoncent d'emblée un texte énigmatique. Toute l'œuvre littéraire et photographique de Cahun cherche à représenter un moi polymorphe et insaisissable. L'autobiographie en question demeure effectivement " non avenue », car elle ne correspond à aucune définition théorique du genre, ni au pacte de Lejeune, ni au " discours en dehors ${ }^{11}$ » de l'autoportrait littéraire selon Michel Beaujour, ni aux théories sur l'autobiographie féminine de diverses féministes américaines, dont Sidonie Smith et Julia Watson ${ }^{12}$, car Cahun refusait d'appartenir à aucun genre ni sexe spécifique :

Brouiller les cartes.

Masculin? Féminin? mais ça dépend des cas. Neutre est le seul genre qui me convienne toujours ${ }^{13}$.

Certains aspects d'Aveux concordent avec la définition de Béatrice Didier du journal intime :

ce type d'écriture ne connaît pas de règle, ni de limites véritables, le journal peut s'ouvrir à n'importe quoi. Tout peut devenir le journal [...]. La page de journal peut devenir une sorte de tableau cubiste, à la Juan Gris, par exemple, où figureraient des collages composés par des morceaux du Petit Parisien [...]. Le dessin, la photographie, les reliques peuvent aussi s'intégrer dans le journal ${ }^{14}$.

Cependant, Aveux n'est pas un journal, mais un livre inclassable, fragmenté et changeant comme les autoportraits photographiques. Les dix photomontages qui l'accompagnent, représentations du corps morcelé lacanien, ou de la tête souvent rasée et démultipliée à l'infini de Cahun, y font écho. C'est un montage verbal de bribes d'aphorismes, de récits de rêves, de fantasmes, de fables, de poèmes, où le sujet en mouvement perpétuel va et vient entre la première et plusieurs troisièmes personnes, entre le féminin et le masculin, le présent et le passé. Cela se lit comme un monologue intérieur, d'un narcissisme à la fois reconnu et remis en question, où s'inscrit un discours d'anorexie, de violence contre le corps du narrateur, ainsi que de rejet de la mère et du féminin en général, éléments qu'on retrouve chez Kay Sage et Unica Zürn :

11. Michel Beaujour, Miroirs d'encre, Paris, Seuil, coll. «Poétique », 1980, p. 7-41.

12. Voir Sidonie Smith et Julia Watson, Women, Autobiography \& Theory: A Reader, Madison, University of Wisconsin Press, 1998, p. 3-52.

13. Claude Cahun, Aveux non avenus [1930], repris dans Écrits, édition présentée et établie par François Leperlier, Paris, Jean-Michel Place, 2002, p. 366.

14. Béatrice Didier, Le Journal intime [1976], Paris, PUF, 1991, coll. « Sup. Littératures modernes », p. 187. 
Je me fais raser les cheveux, arracher les dents, les seins - tout ce qui gêne ou impatiente mon regard - l'estomac, les ovaires, le cerveau conscient et enkysté. [...]

Réalisme.

La mère était si peu appétissante qu'on offrait l'apéritif au nourrisson avant de lui servir le $\operatorname{sein}^{15}$.

En finale, Cahun s'adresse aux lecteurs, avec un détachement de dandy : «Chers Inconnus, gardez bien vos distances: je n'ai que vous au monde ${ }^{16} . »$

Pessimisme, masochisme, misogynie. Comment comprendre ce texte aussi féroce que fragile et à la structure déchiquetée, sans recherches biographiques?

La redécouverte de Claude Cahun, plus de trente ans après sa mort, par François Leperlier s'est faite progressivement, comme en témoignent les nombreuses publications de ce dernier dans un ordre précis. La première monographie biographique de 1992, où l'œuvre photographique et littéraire occupe une place importante ${ }^{17}$, précédait une belle exposition des photographies de Cahun ${ }^{18}$, ainsi que l'édition des Écrits ${ }^{19}$. L'essai de Leperlier ne révélait pas encore le trauma principal à l'origine de l'anorexie et du mal-être de Cahun. À quatre ans, la petite Lucy Schwob fut témoin des premières crises de folie de sa mère, qu'on lui arracha pour l'interner dans un asile psychiatrique. Cette information essentielle paraîtra en 1994, dans un article du catalogue d'une exposition nantaise ${ }^{20}$, où Leperlier dévoile le contenu d'une lettre du 21 janvier 1951 de Cahun à Charles-Henri Barbier. Finalement, en 2006, Leperlier a publié une nouvelle monographie, revue et enrichie ${ }^{21}$. Depuis, l'histoire de Claude Cahun, son roman familial, sa sexualité ambiguë, ainsi que son double talent de photographe et d'écrivaine d'avant-garde, n'ont cessé d'inspirer des travaux de recherche, souvent psychanalytiques, des colloques et des expositions ${ }^{22}$, la dernière ayant eu lieu au Jeu de Paume, du 24 mai au 25 septembre 2011.

15. Claude Cahun, Aveux non avenus, op. cit., respectivement p. 215 et p. 325.

16. Ibid., p. 432.

17. François Leperlier, Claude Cahun : l'écart et la métamorphose, Paris, Jean-Michel Place, 1992.

18. Claude Cahun : photographe, Musée d'art moderne de la ville de Paris, 23 juin-17 septembre 1995.

19. Voir note 13.

20. François Leperlier, "Claude Cahun : la gravité des apparences », dans Le Rêve d'une ville: Nantes et le surréalisme, catalogue de l'exposition au Musée des Beaux-Arts de Nantes, 17 décembre 1994-2 avril 1995, Paris-Nantes, Réunion des musées nationauxMusée des Beaux-Arts de Nantes, p. 260-288.

21. François Leperlier, Claude Cahun : l'exotisme intérieur, Paris, Fayard, 2006.

22. Par exemple Andrea Oberhuber (dir.), Claude Cahun: contexte, posture, filiation, Montréal, Université de Montréal, coll. « Paragraphes », 2007; Louise Downie (dir.), Don't Kiss me: the Art of Claude Cahun and Marcel Moore, Jersey, Heritage Trust, 2000; mes 
Un miroir, réel ou imaginaire, de préférence déformant, demeure une constante dans le corpus littéraire et iconographique de Cahun. Le sujet des autoportraits photographiques, qui change constamment de sexe, se théâtralise, se mécanise en poupée, se dissimule sous des masques, se comprime sous globe, s'emboîte, se cantonne derrière vitres ou barreaux, s'inscrit en trompe-l'œil dans un décor baroque, se reflète dans Aveux. Par ailleurs, Héroïnes $^{23}$, série de contes cruels, fait subir diverses métamorphoses subversives à une sélection de protagonistes féminines de la mythologie grecque, de la Bible ou de contes de fées populaires, ce qui les recrée à l'image de la Claude Cahun sarcastique, sadomasochiste et évasive reflétée dans le spéculum des autoportraits et des premiers textes, dont Aveux. Ces œuvres éclatées, où se manifeste une grande confusion intérieure ont pour source le trauma d'enfance, c'est-à-dire la transformation de la mère en monstre et en paria au sein de la famille, et le déchirement chez la petite fille, tel un morcellement de son corps, lorsqu'on la sépara de cette mère, quelle qu'elle fût.

L'autre long texte autobiographique, inachevé, «Confidences au miroir », fut rédigé en 1945-1946, à un âge mûr, neuf ans avant la mort de Cahun, après un autre trauma extérieur à la famille, qui fit de Cahun et de Malherbe d'authentiques héroïnes. Dès l'invasion nazie de Jersey en 1940, où elles vivaient depuis 1937, elles ont mené seules, « sans relâche [...] des activités de contre-propagande, de démoralisation en direction des troupes d'occupation. Production de tracts, détournement de magazines pronazis, photomontages ${ }^{24} »$.

L'ennemi a longtemps cru à tout un réseau de résistance. Lorsqu'elles furent arrêtées en 1944, envoyées à la prison militaire de l'île et condamnées à mort, tandis que leur maison était perquisitionnée, Cahun, dont la santé fragile supportait difficilement les conditions carcérales, fit une tentative de suicide. Relâchée à la Libération, elle se mit à rédiger ses " Confidences ", un peu comme Carrington vers la même époque écrivit En Bas, pour exorciser son angoisse par rapport à la guerre, au fascisme et à l'enfermement. Ici, le récit se fait à la première personne, identifiée en tant que Lucy Schwob alias Claude Cahun. Si Aveux se présente comme un patchwork, le tissage des « Confidences » crée une chaîne plus ou moins chronologique, constamment interrompue au niveau de la trame par des digressions analeptiques ou proleptiques, récits de rêves et commentaires extradiégétiques de la

articles dans Andrea Oberhuber (dir.), op. cit., dans Mélusine, $\mathrm{n}^{\circ}$ 18, Lausanne (Suisse), L'Âge d'homme, 1998, p. 252-259 et dans S. Smith \& J. Watson (dir.), Interfaces, Ann Arbor, University of Michigan Press, 2002, p. 263-288.

23. Certaines de ces nouvelles ont paru dans Le Mercure de France et Le Journal littéraire en 1925 ; les autres sont restées inédites avant d'être publiées dans les Écrits, op. cit., p. 126-159. Une petite édition autonome, très utile, Claude Cahun, Héroïnes, a été établie par François Leperlier en 2006 chez Mille et une Nuits.

24. François Leperlier, Écrits, op. cit., p. 14. 
narratrice. L'obsession des origines resurgit : «À parents tarés, irresponsablement féconds, mieux vaut enfants morts-nés ${ }^{25}$. " Le titre se réfère à l'absence de miroirs à la prison de Saint-Hélier. En même temps, Cahun se construit un miroir intérieur, correspondant à une forme d'autoanalyse, contrairement à la glace «sans tain » fabriquée dans Aveux, à la manière des surréalistes qu'elle ne connaissait pas encore. On retrouve dans « Confidences » des méditations et des rêves, " miroirs de la réalité », mais Cahun relègue au passé « ce rôle que je m'étais tracé, qui dès lors m'incombait [et qui] n'était tenable qu'en état de transe $»^{26}$. Elle avait trouvé, avant la prison, un réconfort dans la vie utérine à Jersey avec Suzanne son amour, et la mer, « l'unique amant dont les bras nous soient toujours ouverts ${ }^{27} »$. Dans « Confidences », Cahun inscrit un hommage à son ami Desnos (« La liberté l'amour c'est tout un ${ }^{28} \gg$ ) et elle affiche une attitude résolument apolitique. Ce texte inachevé nous transmet ses réflexions de prisonnière. Le temps de l'écriture varie entre un passé récent ou éloigné et le présent, entre histoire et narration. Sa mère, qu'elle appelle Toinette, ponctue le récit, et Cahun ne la rejette plus comme dans Aveux, mais l'évoque avec compassion, en accusant la famille de leur avoir nui à toutes deux. Elle évoque ainsi :

l'obsédante absence de ma mère, traitée en " aliénée », considérée par les siens - par sa mère et sa sœur - comme une honte qu'il convient de cacher $\mathrm{au}$ " monde », le merveilleux et consternant mystère de ce que les adultes nommaient ses « crises », le déchirement de lui être arrachée, rendue pour en être séparée de nouveau ${ }^{29} \ldots$

On apprend que Cahun adulte voyait souvent sa mère, en compagnie de Suzanne et de Marie, sa grand-mère maternelle. Cette mère folle, obèse et optimiste, ayant aussi eu une enfance malheureuse, préférait l'internement à la famille. Surgissent également les souvenirs plus anciens, terrifiants mais poétiques des premières crises :

C'est une statue volante... une frégate glissant sur les nuages... Immense... Mais de cette stature-là, voici qu'elle s'écroule, terrassée... ce n'est plus qu'un gros paquet de linge $[\ldots]$ je m'enfuis, poursuivie par une grande et forte fantômesse, une diablesse blanche ${ }^{30} \ldots$

Cahun croque le portrait d'un père tout en contradictions, en conflit autant avec lui-même qu'avec sa fille. « Confidences » évoque les proches avec plus ou moins d'affection, les surréalistes Breton, Soupault, Aragon, Desnos, d'autres amis, tels Jacques Viot et Henri Michaux.

25. Claude Cahun, «Confidences au miroir » [1945-1946], dans Écrits, op. cit., p. 573.

26. Ibid., p. 573-574.

27. Claude Cahun, Aveux non avenus, ibid., p. 274.

28. Claude Cahun, « Confidences au miroir », op. cit., p. 579.

29. Ibid., p. 585.

30. Ibid., p. 618-619. 
Elle confirme sa complexité sexuelle : « Mon multiple est humain. Un signe hermaphrodite ne suffirait pas à le rendre (à lui rendre justice) », se référant au « miroir rectificatif » ${ }^{31}$ d'une de ses « Héroïnes » de la mythologie grecque, Salmacis, qui devient «Gynandre », une fois attachée à Hermaphrodite comme une sœur siamoise ${ }^{32}$.

Le parcours biographique, psychologique et littéraire de Claude Cahun, de l'adolescence à l'âge mûr, de l'influence du symbolisme de Marcel Schwob à celle du surréalisme d'André Breton, de la haine narcissique à un désir de comprendre autrui, même les parents disparus, ouvre la voie à d'autres explorations herméneutiques, techniques, idéologiques, d'une œuvre dont on ne fait qu'appréhender la richesse.

L'écriture même d'En-Bas (1941-1945), ainsi que son contenu extraordinaire, font que ce récit autobiographique par Leonora Carrington du pire trauma de sa vie se lit comme un roman surréaliste. Jeune artiste et écrivaine, née en 1917, de père anglais et de mère irlandaise, belle, révoltée, passionnée par la peinture, étudiante à l'académie d'Amédée Ozenfant à Londres, Carrington rencontra le surréalisme et l'amour fou en la personne de Max Ernst à un dîner londonien en 1936. Ils devinrent inséparables et malgré les objections paternelles, Carrington quitta sa famille et son pays dès 1937 pour rejoindre Ernst et le groupe surréaliste à Paris. Le couple s'installa ensuite à Saint-Martin d'Ardèche, où ils vécurent trois années paradisiaques à peindre, sculpter et écrire ensemble. Ils y reçurent d'autres surréalistes, tels Roland Penrose et Lee Miller, André Pieyre de Mandiargues et Leonor Fini. La guerre vint briser leur idylle, fin 1939-début 1940. Ernst, d'abord arrêté et incarcéré à Largentière, puis libéré, fut bientôt de nouveau écroué, puis envoyé au camp des Milles en Provence pour étrangers indésirables. Leonora, brutalement privée de son amant et de leur créativité partagée, partit avec une amie en Espagne, où elle sombra dans une dépression nerveuse et fut internée à la clinique psychiatrique des Morales à Santander, à l'instigation de son père et du consul britannique de Madrid. La jeune femme qui se croyait investie d'une mission pour sauver l'Espagne des nazis, fut alors tourmentée et torturée comme une prisonnière de guerre, attachée pendant plusieurs jours sur son lit, où elle croupissait dans ses immondices, violée à l'occasion et forcée de subir des piqûres de cardiazol, drogue qui provoque des convulsions atroces.

Contrairement à Cahun, Carrington n'a pas subi de véritable trauma d'enfance. Cependant, bien qu'aimée de ses parents, elle s'avéra contrariée par l'éducation de sa famille, par son placement dans des établissements scolaires catholiques d'où on la renvoyait immanquablement, et en conflit

31. Ibid., p. 586.

32. Voir mon article «Derrière le Miroir des autoportraits : Hérö̈nes», dans Andrea Oberhuber (dir.), op. cit., p. 125-126. 
perpétuel avec parents et éducateurs, moins par caprice que parce que leur " dressage » traditionnel ne lui correspondait en rien. Seule fille parmi trois frères, elle souffrait de la double mesure entre son éducation et la leur, surtout par rapport à la liberté. Déjà féministe sans le savoir, élevée à la campagne dans divers manoirs du Lancashire, la petite Leonora adorait les animaux, surtout les chevaux auxquels elle s'identifiait et que seules elle et sa mère montaient. Elle appréciait l'excentricité et l'humour de son père Harold Carrington, riche nabab du textile anglais, mais se révoltait contre son autoritarisme patriarcal et victorien. Sa mère irlandaise Mairi Moorhead l'exaspérait par un snobisme nouveau riche, qui l'incita à présenter sa fille contre son gré à la cour de Georges V. Dans un conte fantastique, « La Débutante ${ }^{33}$ ", choisi plus tard par Breton pour son Anthologie de l'humour noir $^{34}$, Leonora s'imagina remplacée par une hyène dévoratrice et malodorante à son bal de débutante, provocant ainsi l'indignation de sa mère. Cette mère demeura néanmoins une alliée, lui apportant son soutien financier pendant les années de bohême avec Max Ernst ${ }^{35}$, et elle lui a légué, avec la grand-mère Moorhead et une nurse également irlandaise, tout un matrimoine de mythologie, de folklore et d'imaginaire celtes, peuplé de créatures hybrides et surnaturelles, qui a nourri sa production littéraire et plastique dès ses jeunes années. C'est par l'intermédiaire de sa mère que Carrington a maintenu un contact familial épisodique, après 1937. À la naissance de son fils aîné Gabriel à Mexico en 1946, sa mère était venue l'assister. La mère n'est jamais mentionnée dans En-Bas, tandis que Carrington découvrait que les psychiatres de Santander, le consul britannique et d'autres employés d'Imperial Chemicals ${ }^{36}$ en Espagne et au Portugal, faisaient partie d'un réseau à la solde de Harold Carrington, chargé d'expédier sa fille gênante en Afrique du Sud.

La force des femmes provient surtout de l'amour de leur mère, qui leur permet d'assumer la maternité à leur tour. Trois suicidées du surréalisme, Frida Kahlo, Kay Sage et Unica Zürn, avaient eu des mères inadéquates, négligentes, voire abusives et ont fini par utiliser leur force potentielle pour se détruire. Carrington par contre, développa très tôt une autonomie et une opiniâtreté rares. Son trauma multiple de 1940, s'enclencha sur un plan personnel, lorsqu'on lui arracha l'homme qu'elle aimait, et se poursuivit avec la réalisation que la guerre l'entourait et qu'elle se trouvait seule sans

33. Leonora Carrington, «La Débutante », écrit vers 1937, publié dans La Dame ovale, avec sept illustrations de Max Ernst, Paris, GLM, 1939. Ce conte a donné son titre au recueil de textes de Carrington établi par Henri Parisot, La Débutante : contes et pièces, Paris, Flammarion, 1978.

34. Le conte de Carrington, « La Débutante », n’est pas présent dans la première édition de 1939. Il ne paraît que dans la deuxième édition de 1947.

35. Voir Salomon Grimberg, catalogue de l'exposition Leonora Carrington What She Might Be, Dallas Museum of Art, 2007-2008, p. 52-54.

36. La compagnie de son père. 
protection, dans un village étranger et hostile, puis avec la trahison de son père et de sa nourrice qu'il avait déléguée à Santander. À chaque étape, elle se comportait en battante, même en plein délire. D'abord seule à SaintMartin : « Je travaillais ma vigne, étonnant les paysans par ma force [...]. Je travaillais aussi mes pommes de terre [...] j'étais d'une force physique que je n'ai connue ni avant, ni après ${ }^{37}$. » Elle avait emporté le passeport d'Ernst en Espagne : «Ce document, qui portait son image, devenait pour moi une entité et, grâce à lui, je conservais mon pouvoir ${ }^{38}$. » Ce pouvoir l'effrayait aussi : «J'étais confiante dans la réussite de ce voyage, mais terriblement angoissée $^{39}$. » Ce mélange d'angoisse et d'illusion d'un pouvoir passant par son corps, ce que Ferenczi appelle un « saut inexplicable dans le corpo$\mathrm{rel}^{40} \gg$, fit qu'elle se croyait responsable d'une mission contre le nazisme : « Madrid était l'estomac du monde et [...] moi j'étais chargée de guérir cet appareil digestif ${ }^{41} »$, situation qui la fit basculer dans un déséquilibre temporaire, univers alternatif et surréaliste, dont elle décryptait les signes et symboles selon son état. Elle s'inventait des rituels pour absorber ses aliments et arrangeait ses articles de toilette dans un ordre qui paraissait alors significatif et alchimique :

j'arrangeais ces objets les uns autour des autres; ils circulaient ensemble sur le chemin céleste, les uns par les autres, formant un rythme complet. Je donnai aux objets, suivant leur emplacement et leur contenu, une vie alchimique $^{42}$.

Pendant sa crise, Carrington s'identifiait à une personnalité plurielle schizophrène : « je sentais que, par le soleil, j’étais androgyne, la lune, le Saint Esprit, une gitane, une acrobate, Leonora Carrington et une femme. Je devais être aussi, plus tard, Elizabeth d'Angleterre ${ }^{43}$ ». S'étant reposée malgré tout en clinique, et ayant mobilisé l'énergie de sa détermination d'accéder au Pavillon «En-Bas », longtemps convoité comme étant « la Terre, le Monde Réel, le Paradis -1 'Éden ${ }^{44} »$, Carrington réussit à guérir, avec l'aide d'un médecin nommé Etcheverria : « j’appris ainsi que le cardiazol était une simple piqûre et non un effet de l'hypnotisme, que Don Luis n'était pas un sorcier mais un bandit, que Covadonga, l'Égypte, Amachu, la Chine [et En-Bas] étaient des pavillons où l'on soignait les fous et qu'il m'en fallait sortir au plus vite ${ }^{45} \gg$. Elle comprit alors que pour devenir une artiste

37. Leonora Carrington, En-bas, Paris, Éric Losfeld, 1973, p. 11.

38. Ibid., p. 12-13.

39. Ibid., p. 14.

40. Sandor Ferenczi, Le Traumatisme, Paris, Payot, coll. «Petite Bibliothèque », 2006, p. 61.

41. Leonora Carrington, En-bas, op. cit., p. 19.

42. Ibid., p. 49.

43. Ibid., p. 47.

44. Ibid.

45. Ibid., p. 63. 
autonome, elle devait quitter Max Ernst et le rôle de «femme-enfant ». Carrington raconta à Marina Warner en 1987, dans un " Postscriptum » à En-Bas, ses retrouvailles avec Ernst à Lisbonne, après avoir échappé à sa gardienne et s'être réfugiée à l'ambassade du Mexique. Elle conclut ainsi :

\footnotetext{
J'étais tourmentée par l'idée que je devais peindre, et lorsque je me trouvai loin de Max [...], je me remis immédiatement à la peinture.

Je ne revis jamais mon père ${ }^{46}$.
}

La jeune artiste s'était donc libérée d'un double surmoi patriarcal. Renée Riese Hubert décrit ainsi la lutte de Carrington : «Au lieu de pleurer Max Ernst, au lieu de s'abandonner à la douleur, elle s'est arrachée presque physiquement à l'emprise de son compagnon ${ }^{47}$. »

En-bas prend la forme d'un double discours, celui du récit autobiographique et celui de l'acte narratif. D'une part, la narratrice/protagoniste/ auteure implicite s'astreint à un genre de pacte autobiographique, d'où sa crainte « de [se] laisser aller à la fiction ${ }^{48}$ », objectif d'autant plus difficile à atteindre que la version citée ici n'était pas la première, qui avait été rédigée en 1941 en anglais, puis perdue. La version française d'En-Bas représente une talking cure, racontée oralement à Mexico en 1943 à un narrataire/ thérapeute, le Docteur Pierre Mabille, dont l'épouse Jeanne Megnen transcrivait et corrigeait le texte. Il s'agit simultanément d'une (auto)-biographie et d'une (auto)-analyse. Carrington commence par dire à Mabille : « Je dois revivre cette expérience, parce que je crois vous être utile en le faisant, et je crois aussi que vous m'aiderez à voyager de l'autre côté de cette frontière en me conservant lucide ${ }^{49}$. $\gg$

Elle établit ainsi une égalité entre eux. La narration se présente comme un journal en trompe-l'œil : le récit aux Mabille se fait en cinq séances, les 23, 24, 25, 26 et 27 août 1943. L'histoire commence à Saint-Martin en mai 1940 avec la deuxième arrestation d'Ernst, mais la durée n'en est pas précisée (quelques semaines, voire deux ou trois mois?). Par ailleurs, la souffrance et l'angoisse décrites à maintes reprises et le désir de les exorciser en les racontant, ôtent toute crédibilité, hormis celle de la projection, à la phrase de Breton dans l'Anthologie de l'humour noir : « Leonora Carrington a gardé la nostalgie des rivages qu'elle a abordés et n'a pas désespéré de les atteindre à nouveau ${ }^{50}$. »

46. Leonora Carrington, «Postscrip. 1987 » [raconté à Marina Warner, juillet 1987, New York], The House of Fear, suivi de Notes from Down Below, New York, E. P. Dutton, 1988, p. 214.

47. Renée Riese Hubert, Magnifying Mirrors: Women, Surrealism, \& Partnership, Lincoln et Londres, University of Nebraska Press, 1994, p. 115-116. Ma traduction.

48. Leonora Carrington, En-bas, op. cit., p. 25.

49. Ibid., p. 9-10.

50. André Breton, Anthologie de l'humour noir [1939], Édition définitive [1966], repris dans Euvres complètes, t. II, Paris, Gallimard, coll. « Bibliothèque de la Pléiade », 1992, 
La psychanalyste Monique Plaza utilise le mot follitérature pour désigner la production privilégiée des moments où la limite entre maladie mentale et créativité, voire génie littéraire, s'estompe chez des auteurs comme Artaud, Carrington ou Duras ${ }^{51}$. On se souviendra du cri d'indignation de Breton contre les psychiatres dans Nadja : «Ils ont enfermé Sade, ils ont enfermé Nietzsche, ils ont enfermé Baudelaire ${ }^{52}$ ! Le poète ne les a pas empêchés d'enfermer Nadja, mais a écrit un livre sur elle, comme elle le lui avait demandé, inscrivant la jeune femme et ses dessins dans l'histoire du surréalisme, même si le texte s'avère plus autobiographique que biographique.

Pour de nombreuses femmes du surréalisme, l'écriture de récits autobiographiques délirants, ou décrivant leurs épisodes de folie de l'intérieur, constituait un acte narratif thérapeutique, un moyen de lutter contre leur détresse psychique, telle une writing cure. Outre aux Aveux et aux « Confidences » de Cahun et à En-Bas de Carrington, ceci peut s'appliquer entre autres à La Cafarde de Bona de Mandiargues (1967), au Journal de Frida Kahlo (écrit entre 1944 et 1954, publié en 1995), aux Eufs de porcelaine de Kay Sage (écrit en 1955, publié en 1996), à L'Homme-Jasmin d'Unica Zürn (1971), ou au Temps n'est rien de Gisèle Prassinos (1958). Or, selon Patrick Mahony, l'autoanalyse de Freud «ne fut pas une cure par la parole mais par l'écrit ${ }^{53} »$; nos femmes surréalistes auraient donc entrepris leur propre « analyse originelle ${ }^{54} »$, terme appliqué à l'autoanalyse freudienne par Octave Mannoni. Freud avait trouvé son référent mythologique originel en CEdipe, tandis que Claude Cahun, en carence de mère, s'est fabriqué des référents féminins multiples, en modifiant à son image et à celles de ses fantasmes révoltés et/ou masochistes des figures mythologiques comme Ève, Dalila, Judith, Pénélope, Hélène, Sapho, Marie, Cendrillon, etc., dans Hérö̈nes. D'autres réinterprétations de mythes surgissent sous la plume ou le pinceau de ces femmes, dont celle de « Léda et le cygne », peint ou photographié d'un point de vue féminin, par Emmy Bridgwater, Mimi Parent et Francesca Woodman ${ }^{55}$ par exemple, constituant autant d'invitations à la critique psychanalytique.

p. 1163. Des deux uniques femmes de l'anthologie, Leonora Carrington et Gisèle Prassinos, seule Prassinos figurait dans la première édition.

51. Voir Monique Plaza, Écriture et folie, Paris, PUF, 1986.

52. André Breton, Nadja [1928], repris dans Euvres complètes, t. I, Paris, Gallimard, coll. « Bibliothèque de la Pléiade », 1988, p. 740.

53. Élisabeth Roudinesco et Michel Plon, Dictionnaire de la psychanalyse, Paris, Fayard, 1997, p. 81. Voir Patrick Mahony, «L'origine de la psychanalyse : la cure par écrit », dans André Haynal (dir.), La Psychanalyse : 100 ans déjà... Contribution à l'histoire intellectuelle du XX siècle, Genève, Georg Éditeur, 1996, p. 155-183.

54. Ibid., p. 82. Voir Octave Manoni, « L'analyse imaginaire » [1967], Clefs pour l'imaginaire ou l'autre Scène, Paris, Seuil, [1969], coll. « Points », 1985, p. 115-130.

55. Voir mon article «Women Artists, Surrealism and Animal Representation », dans le Catalogue de l'exposition Angels of Anarchy/Women Artists and Surrealism, Manchester Art Gallery, 28 septembre 2009-10 janvier 2010, p. 64-73. 
Dire l'importance de la biographie et de la psychanalyse pour mieux comprendre les œuvres féminines du surréalisme ne signifie en aucune façon exclure d'autres approches scientifiques. Au contraire, il s'agit d'une base ou d'un tremplin, pour mettre en valeur une variété d'analyses théoriques. L'évolution des travaux sur Cahun et Carrington va déjà dans ce sens, mais ne fait que commencer en ce qui concerne les autres femmes mentionnées.

Georgiana Colvile

Université Sorbonne Nouvelle - Paris 3, Sorbonne Paris Cité, Centre de Recherche sur le Surréalisme 